\title{
Physical Exercise Potentials Against Viral Diseases Like COVID-19 in the Elderly
}

\begin{abstract}
Sandra Amatriain-Fernández ${ }^{1,2 *}$, Thomas Gronwald ${ }^{3}$, Eric Murillo-Rodríguez ${ }^{4}$, Claudio Imperatori ${ }^{5}$, Alexandre Francisco Solano ${ }^{6}$, Alexandra Latini ${ }^{6+}$ and Henning Budde ${ }^{2 \dagger}$

${ }^{1}$ Faculty of Sport Sciences and Physical Education, University of A Coruña, A Coruña, Spain, ${ }^{2}$ Department of Pedagogy, Faculty of Human Sciences, Medical School Hamburg, Hamburg, Germany, ${ }^{3}$ Department of Performance, Neuroscience, Therapy and Health, Faculty of Health Sciences, Medical School Hamburg, Hamburg, Germany, ${ }^{4}$ School of Medicine, Anahuac Mayab University, Mérida, Mexico, ${ }^{5}$ Cognitive and Clinical Psychology Laboratory, Department of Human Science, European University of Rome, Rome, Italy, ${ }^{6}$ Laboratório de Bioenergética e Estresse Oxidativo - LABOX, Departamento de Bioquímica, Centro de Ciências Biológicas, Universidade Federal de Santa Catarina, Florianópolis, Brazil
\end{abstract}

Keywords: physical exercise, physical activity, viral diseases, coronavirus, COVID-19, immune response, high-risk groups, elderly

\section{OPEN ACCESS}

Edited by:

Graziamaria Corbi,

University of Molise, Italy

Reviewed by:

Carlo Pietro Campobasso,

University of Campania Luigi

Vanvitelli, Italy

Valeria Conti,

University of Salerno, Italy

*Correspondence: Sandra Amatriain-Fernández sandra.amatriain@udc.es

tThese authors share senior authorship

Specialty section:

This article was submitted to Geriatric Medicine,

a section of the journal

Frontiers in Medicine

Received: 07 May 2020 Accepted: 18 June 2020 Published: 03 July 2020

Citation: Amatriain-Fernández S, Gronwald T, Murillo-Rodríguez $E$, Imperatori $C$ Solano AF, Latini $A$ and Budde $H$ (2020) Physical Exercise Potentials

Against Viral Diseases Like COVID-19 in the Elderly. Front. Med. 7:379. doi: 10.3389/fmed.2020.00379

\section{INTRODUCTION}

In the last few months, we have been living through an epic public health threat around the globe due to the spread of a novel coronavirus (SARS-CoV-2) that causes coronavirus disease 2019 or COVID-19 (1). COVID-19 is clinically characterized by fever, cough, fatigue, incapacity to breathe, pneumonia/other respiratory tract symptoms, kidney failure, neurological symptoms and even death (1-7). Recent publications have shown that COVID-19 impairs immune system response by severely compromising the number and function of T cells, especially Natural Killer (NK) cells, by increasing the levels of blood C-reactive protein (CRP) and pro-inflammatory cytokines and causing atrophy of spleen and lymph nodes, along with reduced lymphocytes in lymphoid organs $(2-7)$. This immune dysregulation had a fatal outcome mainly in individuals with pre-existing medical conditions and elderly patients $(2,4,7)$.

According to the United Nations, there were 703 million people aged 65 years or older worldwide in 2019, which implies that about $10 \%$ of our world population is at higher risk for negative prognosis under COVID-19 infection. Aging is characterized by several changes, including exacerbated inflammatory responses mediated by the innate immune system with reduced capacity to protect against infections, cancer and wound healing, leading to more severe consequences of bacterial and viral infections and reduced response to vaccination (8). This pro-inflammatory status renders older individuals susceptible to tissue-damaging immunity and chronic inflammatory diseases. Therefore, interventions that can prevent or retard the decline of immunocompetence would have a considerable clinical and public health impact on this parcel of the population. In this scenario, the regular practice of physical activity and physical exercise has been widely prescribed, including for elderly people, since it favors anti-inflammatory status, promoting healthier aging and reducing all-cause mortality (9).

\section{CHRONIC AND ACUTE PHYSICAL EXERCISE INTERVENTIONS AND THEIR EFFECTS ON THE IMMUNE SYSTEM}

Physical activity is defined as any bodily movement produced by the contraction of skeletal muscles, including sports, leisure activities, dancing, walking, and physical exercise (10). Physical exercise, 
meanwhile, is defined as any planned, structured and purposeful physical intervention. Therefore, physical exercise is physical activity, but physical activity is not necessarily physical exercise (11). Both can be chronic when practiced for a long period of time, or acute when only practiced once (12).

It has been extensively demonstrated that the regular practice of physical exercise (chronic exercise or exercise training) at a moderate intensity [64-76\% of the maximal heart rate (13)] induces the activation of several signaling pathways, conveying a sustained anti-inflammatory and antioxidant response. Some of the positive effects of chronic exercise on the immune system are related to increased $\mathrm{T}$ cell proliferative capacity, neutrophil function, and cytotoxic activity of NK cells (14). For example, 6 months of moderate-intensity aerobic exercise (15-40 min per session, 3 times per week) provoked a significant increase in the $\mathrm{T}$ cell number in the blood of older adults (15); 12 weeks of moderate-intensity walking training (30-40 min, 5 days per week) showed enhanced NK cells activity in elderly women (16); and male volunteers practicing moderate-intensity physical exercise had increased neutrophil phagocytotic activity in an age-associated manner (17). Additionally, chronic exercise has also been shown to enhance the immune response against bacteria and viruses, which might constrain or delay immunological aging or immunosenescence (8). Active muscles release cytokines, which are able to counterbalance pro-inflammatory mediators, i.e., IL$1 \beta$ and IL-18, and stimulate the production of IL-1r $\alpha$ and IL10 interleukins that enhance the anti-inflammatory facet of the immune response (18).

Acute bouts of physical exercise, like a 30-min walk at a moderate intensity (19) or a brief exercise like rapidly ascending 260 stairs (moderate-high intensity) (20) have also been shown to enhance immune system activity by increasing the antipathogen activity of tissue macrophages in parallel with leukocytosis with higher numbers of neutrophils, NK cells, cytotoxic T cells, and immature B cells. Acute bouts of resistance exercises, like $45 \mathrm{~min}$ of a moderate-intensity strength session, showed an increase of the immune system response in aging individuals (21). This kind of exercise that increases muscular strength has been demonstrated to reduce metabolic and cardiovascular diseases and is one of the most important stimuli for fighting osteoporosis in aging individuals (22). This immune-strengthening effect contributes to deconstructing the key pillars of the "open window" theory, which hypothesizes that a single acute and vigorous activity might temporarily impair the immune response, increasing the risk of an opportunistic infection (23). The transient and time-dependent redistribution of immune cells to peripheral tissues might actually represent a heightened state of immunosurveillance and competence driven by a preferential mobilization of cells to areas more susceptible to infection after exercise (e.g., lungs and gut) (24-26).

It has been shown that moderate-intensity cardiovascular exercise executed three times per week for 4 months, prior to viral exposure, improved influenza vaccination responses, with extended duration of antibody levels in older adults (27). These enhanced responses emphasize the importance of exercise during a global pandemic, as it was already suggested (28), since both a single session of acute exercise or a repetition of the exercise over time boosts the immune system independently of age, physical fitness, or the presence of pathologies (29).

Considering that exercise is a drug-free treatment, a specific dosage and administration time for achieving maximal effectiveness will be required (30). The phenomenon called hormesis is defined as an adaptive response of cells and organisms to a moderate (usually intermittent) stress (31). This phenomenon explains both, the benefits that exercise interventions (acute or chronic, with a moderate to high intensity) have in our organism, as well as the negative effects caused by overtraining, like the deregulation of the inflammation processes and a decrease in the ability to maintain homeostasis or homeodynamic regulation (32-34).

\section{PHYSICAL ACTIVITY AND ITS EFFECTS ON THE IMMUNE SYSTEM}

Moderate weekly physical activity (defined as at least $180 \mathrm{~min}$ per week of walking, occupational/volunteer physical activities) was found to correlate with lower levels of inflammatory markers, like the cytokine tumor necrosis factor alpha (TNF- $\alpha$ ) and CRP in a cross-sectional study with a sample of 3,075 subjects aged 7079 years (35). Habitual physical activity was also associated with the maintenance of neutrophil migratory dynamics in a sample of 211 healthy older adults (67 \pm 5 years) (36).

In addition to the benefits of different kinds of physical exercise, it has been shown that a physically active lifestyle might also delay immunosenescence (37), as well as reduce infection risk in the elderly (38). An active lifestyle can also limit adipose tissue accumulation and therefore prevent the development of obesity (39), which represents a state of accelerated aging characterized by low-grade chronic inflammation (37). In fact, the accumulation of visceral fat has been linked to impaired $\mathrm{T}$ cell proliferation and function (40). It is known that adipose tissue suffers a growth in response to prolonged overnutrition, sedentary behavior and aging and turns out to be a major cause of chronic inflammation. The pro-inflammatory status contributes to the onset of damaging diseases such as insulin resistance, diabetes, cardiovascular diseases, musculoskeletal disorders, and some cancers (endometrial, breast, ovarian, prostate, liver, gallbladder, kidney, and colon) (41), which themselves were shown to be risk factors for more severe consequences of the SARS-CoV-2 outbreak (2-7). All of this is relevant especially in light of the affirmed 31 million adults in the US aged 50 or older who are inactive according to the Center for Disease Control and Prevention. The World Health Organization (42) calculates that 1 in 4 adults worldwide does not meet the global recommendations for physical activity per week (a minimum of $150 \mathrm{~min}$ a week of moderate-intensity aerobic activity, or at least $75 \mathrm{~min}$ of vigorous-intensity aerobic physical activity, or an equivalent combination of both). Several interventions have been tried for years to delay aging of the immune system in the elderly with disappointing outcomes, due to high costs of development and administration or for lack of adhesion due to complicated logistics (37). In this scenario, the practice of physical activity 
and physical exercise appears as a potentially cheap and drugfree tool that boosts the immune response without adverse side effects.

\section{MOLECULAR PATHWAYS}

The current understanding of molecular pathways underlying the effects of the regular practice of physical exercise on health includes the activation and interplay of three major systems, namely the immune response, bioenergetics and resistance to oxidative stress [for a review see da Luz Scheffer and Latini (43)]. Sirtuins, a widely distributed family of proteins responsible for the regulation of many fundamental biological processes, including longevity and health span have been suggested to be the master regulators of the beneficial effects of exercise (44). Sirtuins are either mono-ADP ribosyltransferases or nicotinamide adenine dinucleotide (NAD)-dependent histone deacetylases activated by cellular stress, such as that induced by acute or chronic exercise that reactivates cellular defenses and increases cell metabolism and repair-activities (44). Once activated, sirtuins modify histones, transcription factors and cytoplasmic proteins. For example, by deacetylating PGC- $1 \alpha$ (proliferator-activated receptor- $\gamma$ coactivator-1) (45) and the FOXO (class $\mathrm{O}$ of forkhead box) family of transcription factors, sirtuins modulate mitochondrial biogenesis and stimulate the expression of key antioxidant enzymes, including catalase, manganese superoxide dismutase and thioredoxins, respectively (46). In this scenario, it has been demonstrated that exercise increases the activity of sirtuins in the heart and skeletal muscle among other tissues, not only in young people or adults but also in the elderly $(47,48)$. More recently, it was also demonstrated that sirtuins control the production of proinflammatory cytokines in innate immune cells, the type of cells engaged in viral-immune responses. The activation of macrophages, a main source of pro-inflammatory cytokines secreted in response to infection and environmental stress, was shown to occur through two of the major pro-inflammatory pathways in the immune response: NF- $\kappa \mathrm{B}$ and AP-1 pathways $(49,50)$. It was also demonstrated that sirtuins are implicated in the differentiation of activated $\mathrm{T}$ cells into $\mathrm{CD}^{+} \mathrm{T}$ cells, which are lymphocytes responsible for killing host cells infected with pathogens (51). Thus, it seems promising to use physical exercise as a non-pharmacological intervention for increasing resistance to a variety of immune-related diseases.

\section{DISCUSSION}

The aging of the immune system seems to be responsible for several comorbidities presented in the elderly, and $\mathrm{T}$ cells are highly relevant for adaptive immune responses

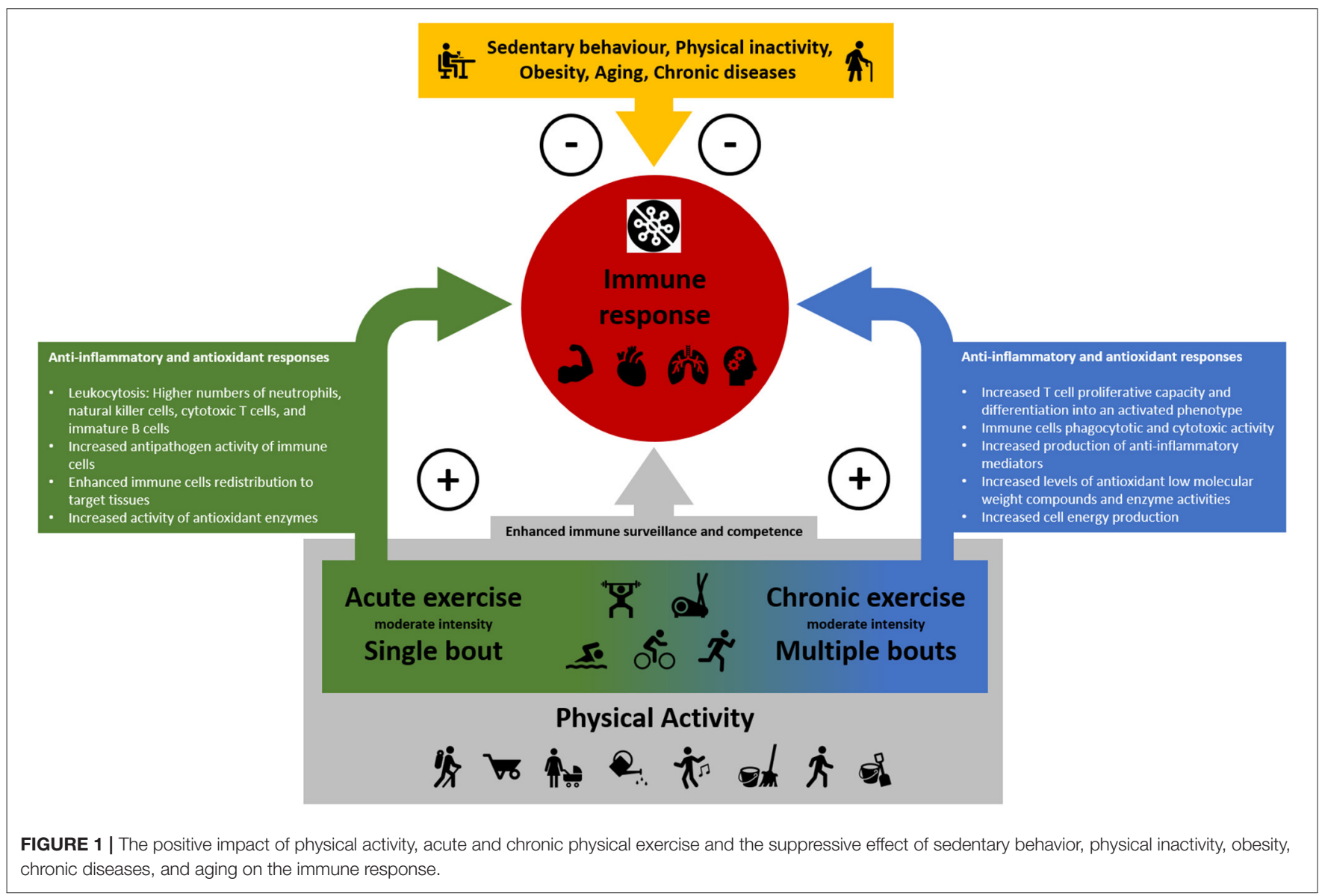


$(8,52)$. Viruses like SARS-CoV-2 can quickly compromise the number and function of $\mathrm{T}$ cells and promote an increased level of pro-inflammatory cytokines in the blood, which might have fatal outcomes in people with pre-existing medical conditions and elderly patients $(2-4,6)$. Thus, both people with existing chronic pathologies and older populations are at higher risk of responding worse to the viral infection due to their higher susceptibility to different infectious diseases, autoimmune diseases, cancer, obesity, and/or a generally sedentary lifestyle. Furthermore, these populations will also have a worse response to vaccination when compared with younger or healthier individuals $(21,25,27,37)$.

The benefits of the physical exercise-induced immune response, including increased antipathogen activity, enhanced recirculation of anti-inflammatory cytokines, and leukocytosis are relevant for fighting viral infections $(14,19,20,53)$. At a molecular level, sirtuins might be one of the key regulators behind the beneficial effects of exercise during the aging process $(34,44)$. Thus, all forms of increased energy expenditure induced by muscle contractions lead to immune-enhancing effects (see

\section{REFERENCES}

1. World Health Organization. Coronavirus Disease (COVID-19) Pandemic. (2020). Available online at: https://www.who.int/emergencies/diseases/novelcoronavirus-2019 (accessed June 25, 2020).

2. Qin C, Zhou L, Hu Z, Zhang S, Yang S, Tao Y, et al. Dysregulation of immune response in patients with COVID-19 in Wuhan, China. Clin Infect Dis. (2020) 12:ciaa248. doi: 10.1093/cid/ciaa248

3. Wang F, Nie J, Wang H, Zhao Q, Xiong Y, Deng L, et al. Characteristics of peripheral lymphocyte subset alteration in COVID-19 pneumonia. I Infect Dis. (2020) 221:1762-9. doi: 10.1093/infdis/jiaa150

4. Chen N, Zhou M, Dong X, Qu J, Gong F, Han Y, et al. Epidemiological and clinical characteristics of 99 cases of 2019 novel coronavirus pneumonia in Wuhan, China: a descriptive study. Lancet. (2020) 395:507-13. doi: 10.1016/s0140-6736(20)30211-7

5. Yang X, Yu Y, Xu J, Shu H, Xia J, Liu H, et al. Clinical course and outcomes of critically ill patients with SARS-CoV-2 pneumonia in Wuhan, China: a single-centered, retrospective, observational study. Lancet Respir Med. (2020) 8:475-81. doi: 10.1016/S2213-2600(20)30079-5

6. Chen G, Wu D, Guo W, Cao Y, Huang D, Wang H, et al. Clinical and immunologic features in severe and moderate Coronavirus Disease 2019. J Clin Investig. (2020) 130:2620-9. doi: 10.1172/JCI1 37244

7. Huang C, Wang Y, Li X, Ren L, Zhao J, Hu Y, et al. Clinical features of patients infected with 2019 novel coronavirus in Wuhan, China. Lancet. (2020) 395:497-506. doi: 10.1016/s0140-6736(20)30183-5

8. Nikolich-Zugich J. The twilight of immunity: emerging concepts in aging of the immune system. Nat Immunol. (2018) 19:109. doi: 10.1038/s41590-017-0006-x

9. Llamas-Velasco S, Villarejo-Galende A, Contador I, Lora Pablos D, Hernández-Gallego J, Bermejo-Pareja F. Physical activity and long-term mortality risk in older adults: A prospective population based study (NEDICES). Prev Med Rep. (2016) 4:546-50. doi: 10.1016/j.pmedr.2016.10.002

10. American College of Sports Medicine. ACSM's Guidelines for Exercise Testing and Prescription. 9th ed. Philadelphia, PA: Lippincott Williams \& Wilkins (2013).

11. Wegner M, Amatriain-Fernández S, Kaulitzky A, Murillo-Rodriguez E, Machado S, Budde H. Systematic review of meta-analyses: exercise
Figure 1). Even if someone were inactive in the past, now might be a good time to start exercising in order to be well-prepared to fight infections. Due to the benefits that an active lifestyle has been shown to have on the immune system, it can be suggested that physically active individuals, including the elderly and people with chronic pathologies, are more likely to have a mild progression of viral diseases like COVID-19.

\section{AUTHOR CONTRIBUTIONS}

SA-F, AL, and HB designed and conceived the paper. SA-F, AL, $\mathrm{HB}, \mathrm{TG}, \mathrm{EM}-\mathrm{R}, \mathrm{CI}$, and AS critically revised the manuscript. All authors contributed to the article and approved the submitted version.

\section{ACKNOWLEDGMENTS}

The authors are grateful to Theodore Griswold for language editing. AL is a CNPq (Conselho Nacional de Desenvolvimento Científico e Tecnológico, Brazil) fellow. effects on depression in children and adolescents. Front Psychiatr. (2020) 11:81. doi: $10.3389 /$ fpsyt.2020.00081

12. Budde H, Schwarz R, Velasques B, Ribeiro P, Holzweg M, Machado S, et al. The need for differentiating between exercise, physical activity, and training. Autoimmun Rev. (2016) 15:110-1. doi: 10.1016/j.autrev.2015.09.004

13. Garber CE, Blissmer B, Deschenes MR, Franklin BA, Lamonte MJ, Lee IM, et al. American college of sports medicine position stand. Quantity and quality of exercise for developing and maintaining cardiorespiratory, musculoskeletal, and neuromotor fitness in apparently healthy adults: guidance for prescribing exercise. Med Sci Sports Exerc. (2011) 43:133459. doi: 10.1249/MSS.0b013e318213fefb

14. Sellami M, Gasmi M, Denham J, Hayes LD, Stratton D, Padulo J, et al. Effects of acute and chronic exercise on immunological parameters in the elderly aged: can physical activity counteract the effects of aging? Front Immunol. (2018) 9:2187. doi: 10.3389/fimmu.2018.02187

15. Woods JA, Ceddia MA, Wolters BW, Evans JK, Lu Q, McAuley E. Effects of 6 months of moderate aerobic exercise training on immune function in the elderly. Mech Ageing Dev. (1999) 109:119. doi: 10.1016/S0047-6374(99)00014-7

16. Nieman DC, Henson DA, Gusewitch G, Warren BJ, Dotson RC, Butterworth DE, et al. Physical activity and immune function in elderly women. Med Sci Sports Exerc. (1993) 25:823-31. doi: 10.1249/00005768-199307000-00011

17. Yan $H$, Kuroiwa A, Tanaka H, Shindo M, Kiyonaga A, Nagayama A. Effect of moderate exercise on immune senescence in men. Eur J Appl Physiol. (2001) 86:105-11. doi: 10.1007/s004210100521

18. Steensberg A, Fischer CP, Keller C, Møller K, Pedersen BK. IL-6 enhances plasma IL-1ra, IL-10, and cortisol in humans. Am J Physiol Endocrinol Metab. (2003) 285:E433-7. doi: 10.1152/ajpendo.00074.2003

19. Nieman DC, Henson DA, Austin MD, Brown VA. Immune Response to a 30-minute walk. Med Sci Sports Exerc. (2005) 37:57-62. doi: 10.1249/01.mss.0000149808.38194.21

20. LaVoy E, Bollard CM, Hanley PJ, Blaney JW, O'Connor DP, Bosch JA, et al. A single bout of dynamic exercise enhances the expansion of MAGE-A4 and PRAME-specific cytotoxic T-cells from healthy adults. Exerc Immunol Rev. (2015) 21:144-53.

21. Bohn-Goldbaum E, Pascoe A, Singh MF, Singh N, Kok J, Dwyer DE, et al. Acute exercise decreases vaccine reactions following influenza vaccination among older adults. Brain BehavImmun Health. (2020) 1:100009. doi: 10.1016/j.bbih.2019.100009 
22. Hong AR, Kim SW. Effects of resistance exercise on bone health. Endocrinol Metab. (2018) 33:435-44. doi: 10.3803/EnM.2018.33.4.435

23. Pedersen BK, Ullum H. NK cell response to physical activity: possible mechanisms of action. Med Sci Sports Exerc. (1994) 26:140-6. doi: 10.1249/00005768-199402000-00003

24. Kruger K, Mooren FC. T cell homing and exercise. Exerc Immunol Rev. (2017) 13:37-54.

25. Simpson RJ. Aging, persistent viral infections, and immunosenescence. Exerc Sport Sci Rev. (2011) 39:23-33. doi: 10.1097/jes.0b013e318201f39d

26. Krüger K, Lechtermann A, Fobker M, Völker K, Mooren FC. Exercise-induced redistribution of $\mathrm{T}$ lymphocytes is regulated by adrenergic mechanisms. Brain Behav Immun. (2008) 22:324-38. doi: 10.1016/j.bbi.2007.08.008

27. Woods JA, Keylock KT, Lowder T, Zelkovich W, Dumich S, Colantuano $\mathrm{K}$, et al. Cardiovascular exercise training extends influenza vaccine seroprotection in sedentary older adults: the immune function intervention trial. J Am Geriatr Soc. (2009) 57:2183-91. doi: 10.1111/j.1532-5415.2009.02563.x

28. Amatriain-Fernández S, Murillo-Rodríguez ES, Gronwald T, Machado S, Budde H. Benefits of physical activity and physical exercise in the time of pandemic. Psychol Trauma. (2020). doi: 10.1037/tra0000643. [Epub ahead of print]

29. Campbell JP, Turner JE. Debunking the myth of exercise-induced immune suppression: redefining the impact of exercise on immunological health across the lifespan. Front Immunol. (2018) 9:648. doi: 10.3389/fimmu.2018.00648

30. Gronwald T, Alves A, Murillo-Rodriguez E, Latini A, Schuette J, Budde H. Standardization of exercise intensity and consideration of a dose-response is essential. Commentary on "exercise-linked FNDC5/irisin rescues synaptic plasticity and memory defects in Alzheimer's models", by Lourenco et al., published 2019 in nature medicine. J Sport Health Sci. (2019) 8:3534. doi: 10.1016/j.jshs.2019.03.006

31. Mattson MP. Hormesis defined. Ageing Res Rev. (2008) 7:17. doi: 10.1016/j.arr.2007.08.007

32. Radak Z, Chung HY, Koltai E, Taylor AW, Goto S. Exercise, oxidative stress and hormesis. Ageing Res Rev. (2008) 7:34-42. doi: 10.1016/j.arr.2007.04.004

33. Billman GE. Homeostasis: the underappreciated and far too often ignored central organizing principle of physiology. Front Physiol. (2020) 11:200. doi: 10.3389/fphys.2020.00200

34. Corbi G, Conti V, Scapagnini G, Filippelli A, Ferrara N. Role of sirtuins, calorie restriction and physical activity in aging. Front Biosci. (2012) 4:76878. doi: $10.2741 / 417$

35. Colbert LH, Visser M, Simonsick EM, Tracy RP, Newman AB, Kritchevsky $\mathrm{SB}$, et al. Physical activity, exercise, and inflammatory markers in older adults: findings from the health, aging and body composition study. J Am Geriatr Soc. (2004) 52:1098-104. doi: 10.1111/j.1532-5415.2004.52307.x

36. Bartlett DB, Fox O, McNulty CL, Greenwood HL, Murphy L, Sapey E, et al. Habitual physical activity is associated with the maintenance of neutrophil migratory dynamics in healthy older adults. Brain Behav Immun. (2016) 56:12-20. doi: 10.1016/j.bbi.2016.02.024

37. Aspinall R, Lang PO. Interventions to restore appropriate immune function in the elderly. Immun Ageing. (2018) 15:5. doi: 10.1186/s12979-017-0111-6

38. Romeo J, Wärnberg J, Pozo T, Marcos A. Physical activity, immunity and infection. Proc Nutr Soc. (2010) 69:390-9. doi: 10.1017/s0029665110001795

39. Higuera-Hernández MF, Reyes-Cuapio E, Gutiérrez-Mendoza M, Barbosa-Rocha N, Barciela-Veras A, Budde $\mathrm{H}$, et al. Fighting obesity: Non-pharmacological interventions. Clin Nutr ESPEN. (2018) 25:50-5. doi: 10.1016/j.clnesp.2018.04.005
40. McLaughlin T, Liu LF, Lamendola C, Shen L, Morton J, Rivas H, et al. T-cell profile in adipose tissue is associated with insulin resistance and systemic inflammation in humans. Arterio Thromb Vasc Biol. (2014) 34:263743. doi: 10.1161/ATVBAHA.114.304636

41. World Health Organization. Obesity and Overweight. (2020). Available online at: https://www.who.int/news-room/fact-sheets/detail/obesity-andoverweight (accessed June 16, 2020).

42. World Health Organization. Global Action Plan on Physical Activity 2018-2030: More Active People for a Healthier World. Geneva: World Health Organization (2019).

43. da Luz Scheffer D, Latini A. Exercise-induced immune system response: Antiinflammatory status on peripheral and central organs. Biochim Biophys Acta Mol Basis Dis. (2020) 1866:165823. doi: 10.1016/j.bbadis.2020.165823

44. Imai SI, Guarente L. It takes two to tango: $\mathrm{NAD}(+)$ and sirtuins in aging/longevity control. NPJ Aging Mech Dis. (2016) 2:16017. doi: $10.1038 /$ npjamd.2016.17

45. Scarpulla RC. Metabolic control of mitochondrial biogenesis through the PGC-1 family regulatory network. Biochim Biophys Acta. (2011) 1813:126978. doi: 10.1016/j.bbamcr.2010.09.019

46. Brunet A. Stress-dependent regulation of FOXO transcription factors by the SIRT1 deacetylase. Science. (2004) 303:2011-5. doi: 10.1126/science.1094637

47. Corbi G, Conti V, Troisi J, Colucci A, Manzo V, Di Pietro P, et al. Cardiac rehabilitation increases SIRT1 activity and $\beta$-hydroxybutyrate levels and decreases oxidative stress in patients with HF with preserved ejection fraction. Oxidative Med Cell Longev. (2019) 2019:7049237. doi: 10.1155/2019/7049237

48. Russomanno G, Corbi G, Manzo V, Ferrara N, Rengo G, Puca AA, et al. The anti-ageing molecule sirt1 mediates beneficial effects of cardiac rehabilitation. Immun Ageing. (2017) 14:7. doi: 10.1186/s12979-017-0088-1

49. Yoshizaki T, Schenk S, Imamura T, Babendure JL, Sonoda N, Bae EJ, et al. SIRT1 inhibits inflammatory pathways in macrophages and modulates insulin sensitivity. Am J Physiol Endocrinol Metab. (2010) 298:E41928. doi: 10.1152/ajpendo.00417.2009

50. Zhang R, Chen HZ, Liu JJ, Jia YY, Zhang ZQ, Yang RF, et al. SIRT1 suppresses activator protein-1 transcriptional activity and cyclooxygenase-2 expression in macrophages. J Biol Chem. (2010) 285:7097-110. doi: 10.1074/jbc.M109.038604

51. Wang R, Green DR. Metabolic checkpoints in activated T cells. Nat Immunol. (2012) 13:907-15. doi: 10.1038/ni.2386

52. Agrawal A, Agrawal S, Gupta S. Dendritic cells in human aging. Exp Gerontol. (2007) 42:421-6. doi: 10.1016/j.exger.2006.11.007

53. Nieman DC, Wentz LM. The compelling link between physical activity and the body's defense system. $J$ Sport Health Sci. (2019) 8:201-17. doi: 10.1016/j.jshs.2018. 09.009

Conflict of Interest: The authors declare that the research was conducted in the absence of any commercial or financial relationships that could be construed as a potential conflict of interest.

Copyright () 2020 Amatriain-Fernández, Gronwald, Murillo-Rodríguez, Imperatori, Solano, Latini and Budde. This is an open-access article distributed under the terms of the Creative Commons Attribution License (CC BY). The use, distribution or reproduction in other forums is permitted, provided the original author $(s)$ and the copyright owner(s) are credited and that the original publication in this journal is cited, in accordance with accepted academic practice. No use, distribution or reproduction is permitted which does not comply with these terms. 